\title{
Aspects of MMOSPA Estimation
}

\author{
David F. Crouse, Student Member, IEEE, Peter Willett, Fellow, IEEE, \\ Yaakov Bar-Shalom, Fellow, IEEE, and Lennart Svensson, Senior Member, IEEE
}

\begin{abstract}
We expand upon existing literature regarding using Minimum Mean Optimal Sub-Pattern Assignment (MMOSPA) estimates in multitarget tracking, noting its advantages in comparison to Maximum Likelihood (ML) and Minimum Mean Squared Error (MMSE) estimation, and look at the practical computation of MMOSPA estimates. We demonstrate the use of MMOSPA estimation in a two-target tracking scenario as well as outside of tracking in a radar angular superresolution scenario.
\end{abstract}

\section{INTRODUCTION}

It has been noted that certain "optimal" tracking algorithms can be "beaten" by approximations [4]. The problem is illustrated in Figure 1, which represents the evolution of particles in a particle filter tracking two targets. Initially, the hypotheses for each of the targets are well separated, but once the targets are closely-spaced for a while, the particles for each target mix, which leads to the MMSE estimates of the target locations being in between both targets. This type of coalescence problem has been extensively studied in the Joint Probabilistic Data Association Filter [6], which approximates joint track hypotheses at each step by a single Gaussian and will be present in any tracker that uses an MMSE estimate for display.

The problem stems from the fact that using the minimum mean squared error estimate for track display can result in track coalescence when there is a high degree of uncertainty in the target identities. Figure 1 shows how identity uncertainty can lead to coalescence. To estimate the states of targets, we shall look at the MOSPA estimate, first considered in [12] and the underlying Optimal Sub-Pattern Assignment Metric (OSPA), introduced in [16], which is very similar to a metric that has been used to measure track accuracy in tracking benchmarks (e.g., [11], [10]). The MMOSPA estimate provides a smooth estimate, that is, it is not subject to the jitter that inherently plagues a ML estimate, but it also avoids the coalescence associated with the MMSE estimate.

In Section [I] we review aspects of MOSPA optimization as applied to full joint target PDFs as well as to particle filter and Gaussian mixture (e.g. the MHT [13] and [2]) approximations of PDFs. We then expand upon this work

This work was partially supported by the Office of Naval Research under contracts N00014-09-10613 and N00014-10-10412.

The first three authors are with the Department of Electrical and Computer Engineering, University of Connecticut, 371 Fairfield Way, U-2157, Storrs, Connecticut 06269 USA (e-mail: \{crouse, willett, ybs\}@engr.uconn.edu). The final author is with the Department of Signals and Systems, Chalmers University of Technology, Göteborg, Sweden (email: lennart.svensson@ chalmers.se).

Portions of this work focussing on tracking, with a more detailed tracking simulation, have been submitted for journal publication [7]

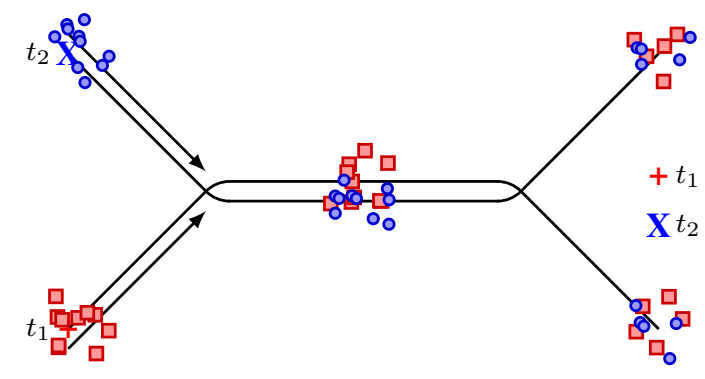

Fig. 1. A diagram showing how the mixing of hypotheses between two targets can lead to MMSE estimates $\left(t_{1}\right.$ and $\left.t_{2}\right)$ that are between the targets.

considering the problem of practical MMOSPA estimation for tracking and provide an optimal solution in the case of a particle filter tracker, demonstrating that the Set JPDAF [18] can be thought of as an approximate MMOSPA tracker. In Section $[\mathrm{IV}$ we use MMOSPA estimation in a tracking scenario as well as in an angular superresolution problem for a linear array, showing that MMOSPA estimation is useful in areas outside of tracking. We conclude in Section $\mathrm{V}$.

\section{THE MOSPA METRIC}

The general notion behind the OSPA metric has existed for many years. For example, a similar measure is defined in [11]. However, a general definition with a rigorous proof that the quantity being discussed was indeed a metric was first given in [16]. This metric is used to evaluate the performance of tracking algorithms without taking into account target labeling.

Suppose that a tracking algorithm outputs $T$ tracks, whereas there is a total of $N_{T}$ true target ${ }^{1}$. Let $\hat{\mathbf{x}}$ be the stacked set of $D$-dimensional state estimates for the tracks and $\hat{\mathbf{x}}$ be the stacked state vectors of the targets at a particular time. Also let $\hat{x}_{t}$ be the state estimate for the th track and $x_{t}$ be the true state of the $t$ th target at time $k$. The OSPA error statistic is given by Equation (1).

The permutation vector a determines the order of the states; its elements consist of a particular permutation of the integers from 1 to $N_{T}$. Thus, $\mathbf{a}$ and $\hat{\mathbf{x}}_{\mathbf{a}}$ can be written as follows:

$$
\begin{aligned}
\mathbf{a} & =\left[a(1), a(2), \ldots, a\left(N_{T}\right)\right]^{\prime} \\
\hat{\mathbf{x}}_{\mathbf{a}} & =\left[\hat{x}_{a(1)}^{\prime}, \hat{x}_{a(2)}^{\prime}, \ldots, \hat{x}_{a\left(N_{T}\right)}^{\prime}\right]^{\prime}
\end{aligned}
$$

\footnotetext{
${ }^{1}$ Though we are discussing this in terms of tracks and truth, the metric may be used with any two sets of objects whose ordering may be changed.
} 


$$
\bar{d}^{(c)}(\hat{\mathbf{x}}, \mathbf{x}) \triangleq \begin{cases}\left(\frac{1}{T} \min _{\mathbf{a}} \sum_{t=1}^{N_{T}} d^{(c)}\left(\hat{x}_{t}, x_{a(t)}\right)^{p}+c^{p}\left(T-N_{T}\right)\right)^{1 / p} & \text { if } N_{T} \leq T . \\ \left(\frac{1}{N_{T}} \min _{\mathbf{a}} \sum_{t=1}^{T} d^{(c)}\left(\hat{x}_{a(t)}, x_{t}\right)^{p}+c^{p}\left(N_{T}-T\right)\right)^{1 / p} & \text { if } N_{T}>T\end{cases}
$$

The permutation change can also be denoted using a permutation matrix, $\chi$, instead of a subscript $2^{2}$ The permutation matrix times the vector puts the vector elements in the desired order. This is the notation used in Appendix 1 .

The variable $p$ is an arbitrary number larger than one. The distance metric $d^{(c)}$ is defined to be

$$
d^{(c)}\left(\hat{x}_{a(t)}, x\right)=\min \left[c, d\left(\hat{x}_{a(t)}, x_{t}\right)\right]
$$

where $d$ is an arbitrary distance metric. We shall use the distance metric that has been most commonly used in previous work, namely, $d\left(\hat{x}_{a(t)}, x_{t}\right)=\left\|\hat{x}_{a(t)}-x_{t}\right\|^{2}$. The value $c$ is a cutoff for the maximum allowable error added by a single track ${ }^{3}$ Basically, this metric tries to find the best assignment of targets to tracks, capping the maximum allowed error and penalizing mismatches in the number of targets to tracks with the maximum possible error for each difference. The expected value of this metric is the MOSPA error.

For the purposes of discussing optimization in this paper, we shall use a specific version of this metric for optimization, the same version that is used in [12]. For our purposes, $T=$ $N_{T}, p=1, d$ will be the $l_{2}$ norm squared, as previously mentioned, and $c \rightarrow \infty$ (since $T-N_{T}=0$, the $c^{p}$ term is not present in the limit). This gives us a metric of

$\bar{d}(\hat{\mathbf{x}}, \mathbf{x})=\frac{1}{N_{T}} \min _{\mathbf{a}} \sum_{t=1}^{N_{T}}\left\|\hat{x}_{t}-x_{a(t)}\right\|^{2}=\frac{1}{N_{T}} \min _{\mathbf{a}}\left\|\mathbf{x}_{\mathbf{a}}-\hat{\mathbf{x}}\right\|^{2}$

The MOSPA error of an estimate whereby the underlying PDF is $p(\mathbf{x})$ is simply the expected value of the OSPA error in (5):

$$
\begin{aligned}
d(\hat{\mathbf{x}}) & \triangleq \frac{1}{N_{T}} \mathrm{E}\left[\min _{\mathbf{a}}\left\|\mathbf{x}_{\mathbf{a}}-\hat{\mathbf{x}}\right\|^{2}\right] \\
& =\frac{1}{N_{T}} \int_{\mathbf{x}} \min \left(d_{1}, d_{2} \ldots d_{N !}\right) p(\mathbf{x}) d \mathbf{x}
\end{aligned}
$$

where

$$
d_{i} \triangleq\left\|\mathbf{x}_{\mathbf{a}_{i}}-\hat{\mathbf{x}}\right\|^{2}=\left\|\mathbf{x}-\hat{\mathbf{x}}_{\mathbf{a}_{i}}\right\|^{2}
$$

The MMOSPA estimate is by definition

$$
\hat{\mathbf{x}}_{M} \triangleq \arg \min _{\hat{\mathbf{x}}} \mathrm{E}\left[\min _{\mathbf{a}}\left\|\mathbf{x}_{\mathbf{a}}-\hat{\mathbf{x}}\right\|^{2}\right]
$$

${ }^{2}$ For example, for two targets, the two possible permutation matrices are

$$
\chi_{1}=\left[\begin{array}{ll}
\mathbf{I}_{D} & \mathbf{0}_{D} \\
\mathbf{0}_{D} & \mathbf{I}_{D}
\end{array}\right] \quad \chi_{2}=\left[\begin{array}{ll}
\mathbf{0}_{D} & \mathbf{I}_{D} \\
\mathbf{I}_{D} & \mathbf{0}_{D}
\end{array}\right]
$$

where $\mathbf{I}_{D}$ is the $D \times D$ identity matrix and $\mathbf{0}_{D}$ is a $D \times D$ matrix of zeros.

${ }^{3}$ This cutoff, in effect, keeps a single lost track from masking the performance of the tracker on all other tracks. It is also the penalty added for having the wrong number of targets and/or tracks.
In general, no explicit formulation for the MMOSPA error estimate exists. However, in [8], an explicit formulation was found for the case where $N_{T}=2$ and the target states are scalar. In Appendix I] we show that this can be generalized to an arbitrary number of scalar targets.

It has been shown that the MMOSPA error estimate in (9) is equivalent to finding the expected value of $\mathrm{x}$ over a "folded" version of $p(\mathbf{x})$ [12]. As a result, the problem of "symmetric" hypotheses causing track coalescence is largely avoided.

\section{PRACTICAL MMOSPA OPTIMIZATION}

We shall prove that the algorithm used to generate the merged state in the Set JPDAF [18] is an approximation to the MMOSPA estimate when used to generate estimates of the target locations for display in the MHT. We will also prove that the expression is exact in the particle filter case, and highlight the analogy between the MMOSPA and the MMSE estimates.

Let us assume that the PDF of the target states at a particular time can be decomposed across $N_{H}$ global hypotheses; the $i$ th hypothesis shall be designated by $u_{i}$ and have probability $w_{i}$. In the MHT, the $u_{i}$ s would represent the individual Gaussian PDFs in a Gaussian mixture, whereas in a particle filter, these would be delta functions representing the individual particles. To derive an approximation to the MMOSPA estimate, we shall use the following definition of an unordered joint covariance matrix ${ }^{4}$ for a particular estimate $\hat{\mathbf{x}}_{M}$,

$$
\begin{gathered}
\mathbf{P} \triangleq \mathrm{E}\left[\min _{\mathbf{a}}\left(\mathbf{x}_{\mathbf{a}}-\hat{\mathbf{x}}_{M}\right)\left(\mathbf{x}_{\mathbf{a}}-\hat{\mathbf{x}}_{M}\right)^{\prime}\right] \\
=\sum_{i=1}^{N_{H}} w_{i} \int_{\mathbf{x}} \min _{\mathbf{a}}\left\{\left(\mathbf{x}_{\mathbf{a}}-\hat{\mathbf{x}}_{M}\right)\left(\mathbf{x}_{\mathbf{a}}-\hat{\mathbf{x}}_{M}\right)^{\prime}\right\} p\left(\mathbf{x} \mid u_{i}\right) d \mathbf{x} \\
\approx \sum_{i=1}^{N_{H}} w_{i} \min _{\mathbf{a}_{i}} \mathrm{E}\left[\left(\mathbf{x}_{\mathbf{a}_{i}}-\hat{\mathbf{x}}_{M}\right)\left(\mathbf{x}_{\mathbf{a}_{i}}-\hat{\mathbf{x}}_{M}\right)^{\prime} \mid u_{i}\right]
\end{gathered}
$$

Note that the minimization operator in $(10)$ is a slight abuse of notation in that we are actually minimizing the trace of the argument rather than the argument itself, which is a matrix. The approximation in (12) decouples the $\mathrm{x}$ between conditional expected values and sets a single ordering for each hypothesis rather than for every single point in the integral. In other words, whereas the changes in ordering

\footnotetext{
${ }^{4}$ The covariance in 16 is just the usual equation for the covariance of a mixture, except some of the orderings are switched. Thus, this is the covariance of the PDF with the switched orderings. It is always less than or equal to the covariance of the original mixture.
} 
in (11) move individual points, the changes in ordering in (12) move individual PDFs. If the PDFs are "peaky", then this is a good approximation.

As used in the optimization on the right-hand side of 97, the MOSPA error is defined to be

$$
\begin{aligned}
d\left(\hat{\mathbf{x}}_{M}\right) & \triangleq \frac{1}{N_{T}} \mathrm{E}\left[\min _{\mathbf{a}}\left\|\mathbf{x}_{\mathbf{a}}-\hat{\mathbf{x}}_{M}\right\|^{2}\right] \\
& =\frac{1}{N_{T}} \int_{\mathbf{x}} \min _{\mathbf{a}}\left\{\sum_{i=1}^{N_{H}} w_{i}\left\|\mathbf{x}_{\mathbf{a}}-\hat{\mathbf{x}}_{M}\right\|^{2} p\left(\mathbf{x} \mid u_{i}\right)\right\} d \mathbf{x}
\end{aligned}
$$

The MOSPA error is equivalent to $1 / N_{T}$ times the trace of the unordered joint covariance matrix in (10) The optimization for the MMOSPA error is, in general, a difficult problem that must be solved iteratively, utilizing numerical integration [12]. Thus, the SJPDAF utilizes the approximation in (12), moving the minimization from being on every point in every integral to simply being fixed over every integral, to lower the complexity of the filter.

Due to its relationship to the expected value, if we know the correct ordering of all possible $\mathbf{x}$, the is we know the a term in (14) for all $\mathrm{x}$, then we can write the MMOSPA estimate as

$$
\hat{\mathbf{x}}_{M} \triangleq \sum_{i=1}^{N_{H}} w_{i} \hat{\mathbf{x}}_{i, \mathbf{a}_{i}}
$$

(the SJPDAF mean), whereby $\hat{\mathbf{x}}_{i, \mathbf{a}_{i}}$ is the vector of the means of the states of all of the tracks according to the $i$ th hypothesis, and in which the ordering of the targets in the vector is given by $\mathbf{a}_{i}$. We shall also define $\hat{\mathbf{x}}_{i}$ to be the same with the original ordering. The covariance matrix of this estimate is $\mathbf{P}_{i, \mathbf{a}_{i}}$, the covariance of the $i$ th component of the mixture such that the ordering of the targets has been rearranged according to $\mathbf{a}_{i}{ }^{5} \mathrm{By}$ adding and subtracting $\hat{\mathbf{x}}_{i, \mathbf{a}_{i}} \hat{\mathbf{x}}_{i, \mathbf{a}_{i}}^{\prime}$ to each term, we can continue the simplification from (12), as shown below

$$
\mathbf{P}=\sum_{i=1}^{N_{H}} w_{i} \min _{\mathbf{a}_{i}}\left[\mathbf{P}_{i, \mathbf{a}_{i}}+\left(\hat{\mathbf{x}}_{i, \mathbf{a}_{i}}-\hat{\mathbf{x}}_{M}\right)\left(\hat{\mathbf{x}}_{i, \mathbf{a}_{i}}-\hat{\mathbf{x}}_{M}\right)^{\prime}\right]
$$

All together, the SJPDAF uses the state estimates from (15) with $\left\{\mathbf{a}_{1}, \ldots, \mathbf{a}_{N_{H}}\right\}$ chosen to minimize the trace of 116, that is

$$
\begin{aligned}
& \left\{\mathbf{a}_{1}, \ldots, \mathbf{a}_{N_{H}}\right\}=\arg _{\mathbf{a}_{1} \ldots \mathbf{a}_{N_{H}}} \operatorname{tr}[\mathbf{P}] \\
& =\arg \min _{\mathbf{a}_{1} \ldots \mathbf{a}_{N_{H}}} \sum_{i=1}^{N_{H}}\left(w_{i}\left\|\hat{\mathbf{x}}_{i, \mathbf{a}_{i}}-\hat{\mathbf{x}}_{M}\right\|^{2}\right)+C \\
& =\arg \max _{\mathbf{a}_{1} \ldots \mathbf{a}_{N_{H}}} \hat{\mathbf{x}}_{M}^{\prime} \hat{\mathbf{x}}_{M}
\end{aligned}
$$

where $C$ is the weighted sum of the traces of $\mathbf{P}_{i}$ and does not depend upon the ordering. Equation (19) follows by expanding the norm in (18), substituting (15), and noting

\footnotetext{
${ }^{5}$ To put it another way, if $\chi_{i} \hat{\mathbf{x}}_{i}=\hat{\mathbf{x}}_{i, \mathbf{a}_{i}}$, in other words $\chi_{i}$ is a permutation matrix corresponding to the permutation given in $\mathbf{a}_{i}$, then $\mathbf{P}_{i, \mathbf{a}_{i}}=\chi_{i} \mathbf{P}_{i} \chi_{i}^{\prime}$.
}

that the quantity $\sum_{i=1}^{N_{H}} w_{i} \hat{\mathbf{x}}_{i, \mathbf{a}_{i}}^{\prime} \hat{\mathbf{x}}_{i, \mathbf{a}_{i}}$ does not depend upon the target ordering. In Appendix $\Pi$, we prove that this optimization is equivalent to a multiframe assignment problem (also known as an S-D assignment problem), as well as a quadratic semi-assignment problem. Additionally, we shall note that the optimization can be formulated explicitly as a quadratic programming problem with $N_{T} ! N_{H}$ terms as described in [17]. In Subsection III-B we present a simple, greedy solution to the problem.

In summary, we can approximate the MMOSPA estimate for the targets by determining the ordering $\mathbf{a}_{1} \ldots \mathbf{a}_{N_{H}}$ that minimizes (19) where our final estimate is given by (15). Each state estimate has an unordered covariance matrix given by one of the diagonal blocks from (16). This covariance matrix worked well in the SJPDAF [18], [6].

Other methods for estimating the target locations from a posterior PDF without causing coalescence, i.e., the problem in Figure 1. have been considered in past work. For example, a method of decomposing an arbitrary two-target PDF into strictly permutation variant and permutation invariant components was introduced in [3] and generalized to an arbitrary number of targets is [8]. The mean of the strictly permutation variant component has many of the properties of the MMOSPA estimate. Similarly, in [9] an approximation to the MMOSPA estimate for an arbitrary PDF was derived for the case wherein the OSPA metric used was $d\left(\hat{x}_{a(t)}, x_{t}\right)=$ $\left\|\hat{x}_{a(t)}-x_{t}\right\|^{4}$ (the $l_{2}$-norm to the fourth, rather than squared).

Note that though the MMOSPA estimate says where targets are, it says nothing about the identities of the targets.

\section{A. Optimality for the Particle Filter}

We shall show that the formulation in (12) is exact in the particle filter case, and thus, the optimization of the simplified cost function in (19) gives us the exact MMOSPA estimate. In this instance, the "hypotheses" consist of $N_{H}$ particles, meaning that $p\left(\mathbf{x} \mid u_{i}\right)$ is a delta function and here $u_{i}$ is conditioning on which particle is "true". Let us designate the location of the delta function corresponding to $u_{i}$ as $\hat{\mathbf{x}}_{i}$. We can thus rewrite the MOSPA error in (14) as

$$
\begin{aligned}
d\left(\hat{\mathbf{x}}_{M}\right) & =\frac{1}{N_{T}} \int_{\mathbf{x}} \min _{\mathbf{a}}\left\{\left\|\mathbf{x}_{\mathbf{a}}-\hat{\mathbf{x}}_{M}\right\|^{2}\right\} \sum_{i=1}^{N_{H}} w_{i} \delta\left[\hat{\mathbf{x}}_{i}-\mathbf{x}\right] d \mathbf{x} \\
& =\frac{1}{N_{T}} \sum_{i=1}^{N_{H}} w_{i} \min _{\mathbf{a}_{i}}\left\{\left\|\hat{\mathbf{x}}_{i, \mathbf{a}_{i}}-\hat{\mathbf{x}}_{M}\right\|^{2}\right\}
\end{aligned}
$$

The simplification from (20) to 21) comes naturally, assuming that no two particles are identical within a permutation of the targets. If that is not the case, it can be shown that there is no advantage to assigning such particles different orderings (i.e. it makes the MOSPA error larger), so (21) still holds. It can be seen that the trace of $(12)$ is equal to 21], so the solution is exact. The same conclusion can also be inferred from the work in [12] 


\section{B. A Greedy Solution}

The optimization in 19 is quite complex. A sequential method of performing the optimization was presented in [18]. We shall give a simple, alternate approach.

Suppose that we have determined the optimal ordering of hypotheses 1 though $N_{H}-1$. Let $\hat{\mathbf{x}}_{\text {sum }}$ be the partial sum of terms $i=1$ through $i=N_{H}-1$ in (15), having weight $w_{\text {sum }}=\sum_{i=1}^{N_{H}-1} w_{i}$. Let $\hat{\mathbf{x}}_{N_{H}}$, a be the last term in the sum in (15), having weight $w_{N_{H}}$. The optimal ordering of the last term according to (19) after discarding constant terms is

$$
\mathbf{a}_{\text {opt }}=\arg \max _{\mathbf{a}}\left\{\hat{\mathbf{x}}_{\text {sum }}^{\prime} \hat{\mathbf{x}}_{N_{H}, \mathbf{a}_{2}}\right\}
$$

This is a two-dimensional assignment problem. We are assigning each state in $\hat{\mathbf{x}}_{N_{H}}$ to one in $\hat{\mathbf{x}}_{\text {sum. }}$. The cost of assigning state vector $i$ in $\hat{\mathbf{x}}_{\text {sum }}$ to state vector $j$ in $\hat{\mathbf{x}}_{N_{H}}$ is $\hat{\mathbf{x}}_{\text {sum }}(i)^{\prime} \hat{\mathbf{x}}_{N_{H}}(j)$. For example, for three targets, we have a cost matrix of the form

$$
A=\left[\begin{array}{lll}
\hat{\mathbf{x}}_{\text {sum }}(1)^{\prime} \hat{\mathbf{x}}_{N_{H}}(1) & \hat{\mathbf{x}}_{\text {sum }}(1)^{\prime} \hat{\mathbf{x}}_{N_{H}}(2) & \hat{\mathbf{x}}_{\text {sum }}(1)^{\prime} \hat{\mathbf{x}}_{N_{H}}(3) \\
\hat{\mathbf{x}}_{\text {sum }}(2)^{\prime} \hat{\mathbf{x}}_{N_{H}}(1) & \hat{\mathbf{x}}_{\text {sum }}(2)^{\prime} \hat{\mathbf{x}}_{N_{H}}(2) & \hat{\mathbf{x}}_{\text {sum }}(2)^{\prime} \hat{\mathbf{x}}_{N_{H}}(3) \\
\hat{\mathbf{x}}_{\text {sum }}(3)^{\prime} \hat{\mathbf{x}}_{N_{H}}(1) & \hat{\mathbf{x}}_{\text {sum }}(3)^{\prime} \hat{\mathbf{x}}_{N_{H}}(2) & \hat{\mathbf{x}}_{\text {sum }}(3)^{\prime} \hat{\mathbf{x}}_{N_{H}}(3)
\end{array}\right]
$$

We have to choose exactly one element in each row and each column. This may be solved using the auction or JVC algorithms [15], or simply by trying all $N_{T}$ ! possible assignments. Thus, we can sequentially determine the optimal orderings in 19 as follows:

1) Fix the ordering of the first hypothesis. Set $i=2$.

2) Determine the ordering for the $i$ th hypothesis, assuming the orderings for hypotheses less than $i$ are correct, by solving the optimization problem in 22 as if $N_{H}=i$.

3) Increment $i$. If $i>N_{H}$, stop; otherwise, go to step 2 .

\section{Simulation Examples}

\section{A. A Tracking Example}

To consider the improvement offered from using MMOSPA estimates in tracking, we ran an MHT using the approximate MMOSPA estimate for Gaussian mixtures, as described in Section [I] the MMSE estimate and the ML estimate to determine the position of two targets at each step.

Two targets moved on trajectories from left to right that approached within $40 \mathrm{~m}$, traveled parallel to each other, and separated, similar to the scenario in Figure 1 . The angle with respect to the horizontal axis at which the targets approached was $\pm 0.6 \mathrm{rad}$. The distance covered by the targets before and after the turns was $800 \mathrm{~m}$. The targets were together for a period of $2 \mathrm{~km}$. The targets moved at a constant speed of $100 \mathrm{~m} / \mathrm{s}$. The targets changed direction according to a coordinated turn model at an angular velocity of $\pm 0.3 \mathrm{rad} / \mathrm{s}$. Observations were made every $\tau=1 \mathrm{~s}$. Track initialization was done for each track by feeding two correctly associated measurements to an Information Filter [1]. One thousand Monte Carlo runs were performed.

Two-dimensional measurements were taken in polar coordinates with $\sigma_{r}=8 \mathrm{~m}$ and $\sigma_{u}=0.52 \mathrm{mrad}$ and converted to Cartesian coordinates. The unbiased conversion method of
[1] was used. A single sensor was placed at $(x, y)$ coordinates of $(1 \mathrm{~km},-40 \mathrm{~km})$. The number of clutter points at each step was determined according to a Poisson process with mean $\lambda=2.179 \times 10^{-6} V$ where $V$ is the area of the surveillance region. Clutter points were placed uniformly over a surveillance region spanning $-1049 \mathrm{~m}<x<30489 \mathrm{~m}$ and $-730 \mathrm{~m}<y<730 \mathrm{~m}$. The average number of clutter measurements per scan was 13 . Both targets had a detection probability of $80 \%$.

The discretized continuous white-noise acceleration model (DCWNA) [1] was used in the trackers. We used a process noise power spectral density of $q_{0}=400 \mathrm{~m}^{2} / \mathrm{s}^{3}$.

The performance of the methods was evaluated by looking at the jitter of the estimates

\begin{tabular}{|c|ccc|}
\hline Metric & ML & MMSE & MOSPA \\
\hline \hline Jitter & 683 & 502 & 493 \\
\hline
\end{tabular}

We can see that MMOSPA estimation provides the lowest level of jitter.

\section{B. Radar Superresolution}

Consider a linear array receiving a reflected signal from two far-field targets (a discussion on angular superresolution is given in [19]). The complex signal received by the $i$ th antenna is

$$
z_{i}=\sum_{m=1}^{2} b_{m} e^{-j \omega x_{i} u_{m}}+w_{i}
$$

where $x_{i}$ is the location of the $i$ th antenna along the linear array, $w_{i}$ is noise, $b_{m}$ is the complex amplitude and $u_{m}$ is a value between -1 and 1 representing the cosine of the angle of arrival of the return from the $m$ th target 7 We shall let $\mathbf{z}$ be the vector of returns from all of the antennas. $\mathbf{a}\left(u_{m}\right)$ shall be a steering vector such that the $i$ th element is

$$
a_{i}\left(u_{m}\right)=e^{-j \omega x_{i} u_{m}}
$$

Assuming that the noise is Gaussian, the PDF of $\mathbf{z}$ is given in 27), where $\mathbf{Q}$ is the noise covariance, which in this simulation we shall take to be $0.5 \mathrm{I}$. We would like to estimate the directions of arrival of the two waves. In this example, we will assume that $b_{1}=b_{1}=1$ and are known. We will place the targets at $u_{1}=-0.3$ and $u_{2}=0.1$, assume that $\omega=2 \pi$ and place the array elements at $x=\{-1,-0.5,0,0.5,1\}$. The prior probability of the angles shall be uniformly distributed 8 Using Bayes' rule we can write

$$
f\left(u_{1}, u_{2} \mid \mathbf{z}\right)=\frac{p\left(\mathbf{z} \mid u_{1}, u_{2}\right) p\left(u_{1}, u_{2}\right)}{p(\mathbf{z})}
$$

\footnotetext{
${ }^{6}$ Two definitions of track jitter are given in [10]. We shall use the second definition of the metric, since it is also commonly used to assess the accuracy of trackers when truth data is not available.

$$
\mathrm{Jitter} \triangleq \sqrt{\frac{1}{N_{\text {runs }}} \sum_{m=1}^{N_{\text {runs }}} \sum_{k=1}^{N}\left\|H \hat{\mathbf{x}}^{m}(k \mid k)-H \hat{\mathbf{x}}^{m}(k \mid k-1)\right\|^{2}}
$$

${ }^{7}$ To get a unique solution, we assume that the target is in front of the linear array.

${ }^{8}$ Thus $p\left(u_{1}, u_{2}\right)=1 / 4$ if $-1 \leq\left\{u_{1}\right.$ and $\left.u_{2}\right\} \leq 1$.
} 


$$
p\left(\mathbf{z} \mid u_{1}, u_{2}\right)=|\pi \mathbf{Q}|^{-1} \exp \left\{-\left[\mathbf{z}-\mathbf{a}\left(u_{1}\right) b_{1}-\mathbf{a}\left(u_{2}\right) b_{2}\right]^{H} \mathbf{Q}^{-1}\left[\mathbf{z}-\mathbf{a}\left(u_{1}\right) b_{1}-\mathbf{a}\left(u_{2}\right) b_{2}\right]\right\}
$$

where

$$
p(\mathbf{z})=\frac{1}{4} \int_{-1}^{1} \int_{-1}^{1} p\left(\mathbf{z} \mid u_{1}, u_{2}\right) d u_{1} d u_{2}
$$

Using the results of Appendix [1] an explicit solution for the MMOSPA estimate of the angles of arrival of the two targets is given by

$$
\hat{\mathbf{u}}_{M}=2 \frac{\int_{-1}^{1} \int_{-1}^{u_{1}}\left[\begin{array}{l}
u_{1} \\
u_{2}
\end{array}\right] p\left(\mathbf{z} \mid u_{1}, u_{2}\right) d u_{2} d u_{1}}{\int_{-1}^{1} \int_{-1}^{1} p\left(\mathbf{z} \mid u_{1}, u_{2}\right) d u_{1} d u_{2}}
$$

The 2 comes from the fact that both permutations are the same.

Running the simulation with a noiseless measurement, we get the correct estimates for both of the angles of arrival, whereas the MMSE estimates are -0.1 for both targets $-\mathrm{a}$ point that lies between the angles- and are useless. Running the simulation with noise generated according to the model for 500 Monte Carlo runs, we get a MOSPA error of 0.006 for the estimates of $\mathbf{u}$.

\section{CONCLUSION}

We reviewed literature regarding the exact computation of the MMOSPA estimate in the particle filter and an approximation in an MHT, expanding upon previous work to show the relationship between the solution and the S-D assignment and quadratic semi assignment problems and provide an explicit solution for scalar states. We then demonstrated that MMOSPA estimation can be used to reduce jitter in track display, as well as to provide useful estimates in radar superresolution scenarios where MMSE estimation fails.

\section{REFERENCES}

[1] Y. Bar-Shalom, X. R. Li, and T. Kirubarajan, Estimation with Applications to Tracking and Navigation. New York: John Wiley \& Sons, Inc., 2001.

[2] S. Blackman, "Multiple hypothesis tracking for multiple target tracking," IEEE Aerospace and Electronic Systems Magazine, vol. 19, no. 1, pp. 5-18, Jan. 2004.

[3] H. A. P. Blom and E. A. Bloem, "Permutation invariance in Bayesian estimation of two targets that maneuver in and out [of] formation flight," in Proceedings of the 12th International Conference on Information Fusion, Seattle, WA, Jul. 2009, pp. 1296-1303.

[4] H. A. P. Blom, E. A. Bloem, Y. Boers, and H. Driessen, "Tracking closely spaced targets: Bayes outperformed by an approximation?" in Proceedings of the 11th International Conference on Information Fusion, Cologne, Germany, Jul. 2008.

[5] R. E. Burkard, E. Çela, P. M. Pardalos, and L. S. Pitsoulis, The Quadratic Assignment Problem. Kluwer Academic Publishers, 1998, vol. 3, pp. 241-338.

[6] D. F. Crouse, Y. Bar-Shalom, P. Willett, and L. Svensson, "The JPDAF in practical systems: Computation and snake oil," in Proceedings of SPIE: Signal and Data Processing of Small Targets Conference, vol. 7698, Apr. 2010.

[7] D. F. Crouse, P. Willett, and Y. Bar-Shalom, "Developing a real-time track display that operators do not hate," IEEE Transactions on Signal Processing, submitted June 2010.

[8] — - "Generalizations of Blom and Bloem's PDF decomposition for permutation-invariant estimation," in Proceedings of the IEEE International Conference on Acoustics, Speech, and Signal Processing, May 2011.
[9] D. F. Crouse, P. Willett, M. Guerriero, and L. Svensson, "An approximate minimum MOSPA estimator," in Proceedings of the IEEE International Conference on Acoustics, Speech, and Signal Processing, May 2011.

[10] O. E. Drummond, "Best hypothesis target tracking and sensor fusion," in Proceedings of SPIE: Signal and Data Processing of Small Targets Conference, vol. 3809, Denver, CO, Oct. 1999, pp. 586-600.

[11] B. E. Fridling and O. E. Drummond, "Performance evaluation methods for multiple target tracking algorithms," in Proceedings of SPIE: Signal and Data Processing of Small Targets Conference, vol. 1481, Aug. 1991, pp. 371-383.

[12] M. Guerriero, L. Svensson, D. Svensson, and P. Willett, "Shooting two birds with "two" bullets: How to find minimum mean OSPA estimates," in Proceedings of the 13th International Conference on Information Fusion, Edinburgh, Scotland, Jul. 2010.

[13] W. Koch, "Advanced target tracking techniques," in Advanced Radar Signal and Data Processing, ser. Educational Notes RTO-EN-SET086, Paper 2, Neuilly-sur-Seine, France, 2006, pp. 2.1-2.34.

[14] E. M. Loiola, N. M. Maia de Abreu, P. O. Boaventura-Netto, P. Hahn, and T. Querido, "A survey for the quadratic assignment problem," European Journal of Operational Research, vol. 176, no. 2, pp. 657690, Jan. 2007.

[15] K. R. Pattipati, R. L. Popp, and T. Kirubarajan, "Survey of assignment techniques for multitarget tracking," in Multitarget-Multisensor Tracking: Applications and Advances, Y. Bar-Shalom and W. D. Blair, Eds. Boston: Artech House, 2000, vol. III, ch. 2.

[16] D. Schuhmacher, B.-T. Vo, and B.-N. Vo, "A consistent metric for performance evaluation of multi-object filters," IEEE Transactions on Signal Processing, vol. 56, no. 8, pp. 3447-3457, Aug. 2008.

[17] D. Svensson, "Target tracking in complex systems," Ph.D. dissertation, Chalmers University of Technology, Göteborg, Sweden, 2010, pp. $111-146$

[18] L. Svensson, D. Svensson, and P. Willett, "Set JPDA algorithm for tracking unordered sets of targets," in Proceedings of the 12th International Conference on Information Fusion, Seattle, WA, Jul. 2009, pp. 1187-1194.

[19] W.-D. Wirth, Radar Techniques Using Array Antennas. London, UK: The Institution of Engineering and Technology, 2008.

\section{APPENDIX I}

\section{AN EXPlicit MMOSPA SOLUTION FOR SCALAR STATES}

In [8], an explicit solution for the MMOSPA estimate of two targets having scalar states was presented. We shall generalize the results to PDFs having an arbitrary number of targets having scalar states.

The MMOSPA solution is not unique, because the ordering of the targets can always be switched. We shall thus define a unique ordering of the elements in the MMOSPA estimate. For $N_{T}$ targets, we shall say that $\hat{x}_{1} \geq \hat{x}_{2} \geq \ldots \geq \hat{x}_{N_{T}}$. Let us consider when each ordering of the measure is the minimum in (7). Suppose that we have costs corresponding to two orderings, $d_{a}$ and $d_{b}$, as defined in (8) that differ only in that targets $x_{i}$ and $x_{j}$ have been swapped. $d_{a}$ is the dominant ordering when

$$
d_{a} \leq d_{b} \Leftrightarrow\|\mathbf{x}-\hat{\mathbf{x}}\|^{2} \leq\|\mathbf{x}-\chi \hat{\mathbf{x}}\|^{2}
$$

where $\chi$ is a permutation matrix such that $\chi \hat{\mathbf{x}}$ switches $\hat{x}_{i}$ and $\hat{x}_{j}$ in $\hat{\mathbf{x}}$. Eliminating terms that are independent of the ordering, noting that $\hat{\mathbf{x}}^{T} \hat{\mathbf{x}}=\hat{\mathbf{x}}^{T} \chi \hat{\mathbf{x}}$, we can simplify 31 to

$$
\mathbf{x}^{T} \hat{\mathbf{x}} \geq \mathbf{x}^{T} \chi \hat{\mathbf{x}}
$$

The vector $\mathbf{x}_{R}$ shall represent the remaining target states whose ordering is the same in $d_{a}$ and $d_{b}$. We will break the 
states into parts that are switched, and everything else that is constant:

$$
\mathbf{x}=\left[\begin{array}{c}
x_{i} \\
x_{j} \\
\mathbf{x}_{R}
\end{array}\right] \quad \hat{\mathbf{x}}=\left[\begin{array}{c}
\hat{x}_{i} \\
\hat{x}_{j} \\
\hat{\mathbf{x}}_{R}
\end{array}\right]
$$

We can see that 32 can be simplified to

$$
\left(x_{i}-x_{j}\right)\left(\hat{x}_{i}-\hat{x}_{j}\right) \geq 0
$$

Assuming, without loss of generality, that $i>j$, this means that

$$
d_{a} \leq d_{b} \Leftrightarrow x_{i} \geq x_{j}
$$

Thus, based on the comparison of pairs, as in (35), we can uniquely determine the correct ordering to be used in (7) When we flip the ordering of two states, we cause multiple points in the PDF to have the same OSPA cost. Keeping this in mind, since we have defined an ordering of the results, we can directly evaluate the MMOSPA estimate from (9):

$$
\hat{\mathbf{x}}_{M}=\int_{-\infty}^{\infty} \int_{-\infty}^{x_{1}} \ldots \int_{\infty}^{x_{N_{T}-1}} \mathbf{x} \sum_{i=1}^{N_{T} !} p\left(\chi_{i} \mathbf{x}\right) d x_{N_{t}} \ldots d x_{2} d x_{1}
$$

\section{APPENDIX II \\ MMOSPA ESTIMATION AS S-D ASSIGNMENT AND QuAdratic SEMI-AssignMENT PROBlEMS}

As explained in Section III, the optimal ordering of the states for calculating the MMOSPA estimate is the following optimization problem 9

$$
\begin{aligned}
\left\{\mathbf{a}_{1}, \ldots, \mathbf{a}_{N_{H}}\right\} & =\arg \max _{\mathbf{a}_{1} \ldots \mathbf{a}_{N_{H}}} \hat{\mathbf{x}}_{M}^{\prime} \hat{\mathbf{x}}_{M} \\
& =\arg \max _{\mathbf{a}_{1} \ldots \mathbf{a}_{N_{H}}} \sum_{i=1}^{N_{H}} \sum_{j=1}^{N_{H}} w_{i} w_{j} \hat{\mathbf{x}}_{i, \mathbf{a}_{i}}^{\prime} \hat{\mathbf{x}}_{j, \mathbf{a}_{j}}
\end{aligned}
$$

This optimization is equivalent to the following quadratic semi-assignment problem

$$
\begin{gathered}
\hat{\chi}=\arg \max _{\chi}\left\{\sum_{k_{1}=1}^{N_{T} !} \sum_{k_{2}=1}^{N_{T} !} \sum_{i=1}^{N_{H}} \sum_{j=1}^{N_{H}} p_{i} p_{j} \hat{\mathbf{x}}_{i, k_{1}}^{\prime} \hat{\mathbf{x}}_{j, k_{2}} \chi_{i, k_{1}} \chi_{j, k_{2}}\right\} \\
\text { such that } \sum_{k=1}^{N_{T} !} \chi_{i, k}=1 \quad \chi_{i, k} \in\{0,1\}
\end{gathered}
$$

In this case $\chi_{i, k}$ is unity if the $k$ th ordering of $\hat{\mathbf{x}}_{i}$ is chosen ${ }^{10}$ thus $\chi$ implicitly defines $\left\{\mathbf{a}_{1}, \ldots, \mathbf{a}_{N_{H}}\right\}$. Since $\hat{\mathbf{x}}$ contains $N_{T}$ target states, there are a total of $N_{T}$ ! possible $k$ values for each $\chi_{i, k}$. In general, quadratic semi-assignment problems are NP-hard [14], [5].

\footnotetext{
${ }^{9} \mathrm{We}$ shall also note that in [17], the optimization problem has been shown to be equivalent to a constrained quadratic programming problem with $N_{T} ! N_{H}$ terms.

${ }^{10}$ Here we assume an arbitrary enumerative mapping between $k$ and the possible ordering of the target states in $\mathbf{x}_{i}$. For example, $k$ might be the index of the lexicographically ordered permutations of the elements of might decide that $\hat{\mathbf{x}}_{i}$
}

The optimization in (38) can also be formulated as an multiframe assignment problem, as used for tracking in $[15]^{11}$ The optimization in (38) can be rewritten as

$$
\left\{\mathbf{a}_{1}, \ldots, \mathbf{a}_{N_{H}}\right\}=\arg \max _{\mathbf{a}_{1} \ldots \mathbf{a}_{N_{H}}} \sum_{i=2}^{N_{H}} w_{i} \hat{\mathbf{x}}_{i, \mathbf{a}_{i}}^{\prime} \sum_{j=1}^{i-1} w_{j} \hat{\mathbf{x}}_{j, \mathbf{a}_{j}}
$$

The simplification comes from eliminating all terms that are independent of the ordering of the states (i.e., $\hat{\mathbf{x}}_{i, \mathbf{a}_{i}}^{\prime} \hat{\mathbf{x}}_{i, \mathbf{a}_{i}}$ terms), grouping all identical products, and eliminating a multiplicative coefficient of 2 .

Define $\hat{\mathbf{x}}_{k}\left(i_{k}\right)$ to be the state of target $i_{k}$ in the $k$ th hypothesis. In other words, we are indexing the state vectors in $\hat{\mathbf{x}}_{k}$. From (41), we can see that

$$
c_{k}\left(i_{1}, \ldots, i_{N_{H}}, n\right)=\operatorname{Pr}\left\{u_{N_{H}}\right\} \hat{\mathbf{x}}_{N_{H}}\left(i_{N_{H}}\right)^{\prime} \sum_{j=1}^{N_{H}-1} w_{j} \hat{\mathbf{x}}_{j}\left(i_{j}\right)
$$

Now, let us define a binary association variable $\rho_{i_{1}, i_{2}, \ldots i_{N_{H}}, n}$ that takes value 1 if $\left(i_{1}, i_{2}, \ldots, i_{N_{H}}\right)$ is associated to track $n$ and value 0 otherwise. The optimal assignments can then be obtained by maximizing the cost

$\hat{\boldsymbol{\rho}}=\arg \max _{\boldsymbol{\rho}} \sum_{n=1}^{N_{T}} \sum_{i_{1}=1}^{N_{T}} \sum_{i_{2}=1}^{N_{T}} \ldots \sum_{i_{N_{H}}=1}^{N_{T}} c_{i_{1}, i_{2}, \ldots, i_{N_{H}}, n} \rho_{i_{1}, i_{2}, \ldots i_{N_{H}}, n}$

subject to the constraints

$$
\begin{aligned}
& \forall \rho \in\{0,1\} \\
& \sum_{i_{1}=0}^{N_{T}} \sum_{i_{2}=0}^{N_{T}} \ldots \sum_{i_{N_{H}}=0}^{N_{T}} \rho_{i_{1}, i_{2}, \ldots i_{N_{H}}, n}=1 \quad n \in 1,2, \ldots, N_{T} \\
& \sum_{n=1}^{N_{T}} \sum_{i_{2}=0}^{N_{T}} \ldots \sum_{i_{N_{H}}=0}^{N_{T}} \rho_{i_{1}, i_{2}, \ldots i_{N_{H}}, n}=1 \quad i_{1} \in 1,2, \ldots, N_{T} \\
& \sum_{n=1}^{N_{T}} \sum_{i_{1}=0}^{N_{T}} \ldots \sum_{i_{N_{H}}=0}^{N_{T}} \rho_{i_{1}, i_{2}, \ldots i_{N_{H}}, n}=1 \quad i_{2} \in 1,2, \ldots, N_{T} \\
& \sum_{n=1}^{N_{T}} \sum_{i_{1}=0}^{N_{T}} \ldots \sum_{i_{N_{H}-1}=0}^{N_{T}} \rho_{i_{1}, i_{2}, \ldots i_{N_{H}}, n}=1 \quad i_{S} \in 1,2, \ldots, N_{T}
\end{aligned}
$$

The $\rho$ are binary association variables such that $\rho_{i_{1}, i_{2}, \ldots i_{N_{H}}, n}=1$ if target $n$ is assigned the positions in the hypotheses defined by the $N_{H}$-tuple $\left\{i_{1}, i_{2}, \ldots i_{N_{H}}\right\}$. For example, if there are 3 hypotheses and two targets, if $\rho_{1,2,1,1}=1$ then that means that target 1 is assigned position 1 in hypotheses 1 and 3 in the sum in Equation 15] and position 2 in hypothesis 2 . From the constraints, we also know that $\rho_{2,1,2,2}=1$ and all other $\rho$ terms must be zero.

\footnotetext{
${ }^{11}$ The multiframe assignment problem is sometimes called the S-D assignment problem, where $\mathrm{S}$ corresponds to $N_{H}$ in our problem. Solutions to the S-D assignment problem have been considered in [15].
} 